\title{
POSTERIOR REGRET $\Gamma$-MINIMAX ESTIMATION IN A NORMAL MODEL WITH ASYMMETRIC LOSS FUNCTION
}

Abstract. The problem of posterior regret $\Gamma$-minimax estimation under LINEX loss function is considered. A general form of posterior regret $\Gamma$ minimax estimators is presented and it is applied to a normal model with two classes of priors. A situation when the posterior regret $\Gamma$-minimax estimator, the most stable estimator and the conditional $\Gamma$-minimax estimator coincide is presented.

1. Introduction. Robust Bayesian analysis is concerned with the effect of changing a prior within a class $\Gamma$ on some quantity, for example: the posterior risk, the Bayes risk, the posterior expected value. But it is interesting not only in investigating the range but also in constructing optimal procedures. There are several concepts of optimal rules: $\Gamma$-minimax rules (e.g. Berger [1]), conditional $\Gamma$-minimax rules (e.g. Betro and Ruggeri [2], Męczarski [6]), the most stable rules (e.g. Męczarski and Zieliński [7], Boratyńska [3]), and the posterior regret $\Gamma$-minimax rules (e.g. Rios Insua and Ruggeri [8]). A brief overview of the $\Gamma$-minimax inference standpoint as a way to select a robust rule is presented by Vidakovic [9].

This paper considers the problem of the posterior regret $\Gamma$-minimax estimation of an unknown real parameter $\theta$ under the asymmetric loss function (LINEX loss)

$$
L(\theta, a)=b[\exp (c(\theta-a))-c(\theta-a)-1],
$$

where $c$ and $b$ are known parameters and $c \neq 0$ and $b>0$ (without loss of generality we will assume $c>0$ and $b=1$ ), when priors belong to a class $\Gamma$.

2000 Mathematics Subject Classification: Primary 62C10; Secondary 62F15.

Key words and phrases: Bayes estimators, classes of priors, robust Bayes estimation, posterior regret $\Gamma$-minimax estimation, LINEX loss. 
For motivation to use LINEX loss see Wan, Zou and Lee [10] and references therein. The posterior regret $\Gamma$-minimax estimation with square loss function is considered in Rios Insua and Ruggeri [8]. The LINEX loss function, the conditional $\Gamma$-minimax estimation and the most stable estimation are considered in Boratyńska [4] and Boratyńska and Drozdowicz [5].

We present a general form of the posterior regret $\Gamma$-minimax estimator of a parameter $\theta$ and apply it to the estimation of the expected value in a normal model with two classes of priors. We show when the estimator obtained coincides with the conditional $\Gamma$-minimax estimator and the most stable estimator presented in [5].

2. Posterior regret $\Gamma$-minimax estimation. Let $X$ be an observed random variable with a distribution $P_{\theta}$ indexed by a real parameter $\theta \in \Theta$, with a density $p_{\theta}(x)$ with respect to some $\sigma$-finite measure $\mu$. Suppose that $\theta$ has a prior distribution $\Pi$ with a density with respect to some $\sigma$-finite measure. If $X=x$ then the posterior risk of an estimator $\hat{\theta}$ under LINEX loss function is equal to

$$
R_{x}(\Pi, \widehat{\theta}(x))=e^{-c \widehat{\theta}(x)} E_{\Pi}\left(e^{c \theta} \mid x\right)-c E_{\Pi}(\theta \mid x)+c \widehat{\theta}(x)-1,
$$

where $E_{\Pi}(g(\theta) \mid x)$ denotes the expected value of a function $g(\theta)$ when $\theta$ has a posterior distribution. The Bayes estimator at a point $x$ is

$$
\widehat{\theta}^{\Pi}(x)=\frac{1}{c} \ln E_{\Pi}\left(e^{c \theta} \mid x\right) .
$$

In the robust Bayesian spirit, assume that we are only able to elicit a class $\Gamma$ of priors. We are interested in calculating the posterior regret $\Gamma$-minimax estimator $\widehat{\theta}_{\mathrm{PR}}$, i.e. the estimator satisfying, for every value $x$ of the random variable $X$,

$$
\sup _{\Pi \in \Gamma} r_{x}\left(\Pi, \widehat{\theta}_{\mathrm{PR}}(x)\right)=\inf _{a \in \mathbb{R}} \sup _{\Pi \in \Gamma} r_{x}(\Pi, a),
$$

where $r_{x}(\Pi, a)$ is the posterior regret equal to

$$
r_{x}(\Pi, a)=R_{x}(\Pi, a)-R_{x}\left(\Pi, \widehat{\theta}^{\Pi}(x)\right) .
$$

Let us recall two other definitions of optimal estimators.

The estimator $\widehat{\theta}_{s}$ of a parameter $\theta$ is called the most stable if $\inf _{a \in \mathbb{R}}\left(\sup _{\Pi \in \Gamma} R_{x}(\Pi, a)-\inf _{\Pi \in \Gamma} R_{x}(\Pi, a)\right)=\sup _{\Pi \in \Gamma} R_{x}\left(\Pi, \widehat{\theta}_{s}(x)\right)-\inf _{\Pi \in \Gamma} R_{x}\left(\Pi, \widehat{\theta}_{s}(x)\right)$ for every value $x$ of the random variable $X$.

The estimator $\widetilde{\theta}$ is called conditional $\Gamma$-minimax if

$$
\inf _{a \in \mathbb{R}} \sup _{\Pi \in \Gamma} R_{x}(\Pi, a)=\sup _{\Pi \in \Gamma} R_{x}(\Pi, \widetilde{\theta}(x))
$$

for every value $x$ of $X$. 
From now on we will suppress $x$ wherever possible in formulas for estimators.

Theorem 1. Let $X=x$. Suppose $\underline{a}=\underline{a}(x)=\inf _{\Pi \in \Gamma} \widehat{\theta}^{\Pi}(x)$ and $\bar{a}=$ $\bar{a}(x)=\sup _{\Pi \in \Gamma} \widehat{\theta}^{\Pi}(x)$ are finite and $\underline{a}<\bar{a}$. Then

$$
\widehat{\theta}_{\mathrm{PR}}=\frac{1}{c} \ln \frac{e^{c \bar{a}}-e^{c \underline{a}}}{c(\bar{a}-\underline{a})}=\underline{a}+\frac{1}{c} \ln \frac{e^{c(\bar{a}-\underline{a})}-1}{c(\bar{a}-\underline{a})}
$$

and

$$
\inf _{a \in \mathbb{R}} \sup _{\Pi \in \Gamma} r_{x}(\Pi, a)=\frac{c(\bar{a}-\underline{a})}{\exp (c(\bar{a}-\underline{a}))-1}-\ln \frac{c(\bar{a}-\underline{a})}{\exp (c(\bar{a}-\underline{a}))-1}-1 .
$$

Proof. For given $X=x$ let $h=h(\Pi, x)=E_{\Pi}\left(e^{c \theta} \mid x\right)$. Then $\widehat{\theta}^{\Pi}=\frac{1}{c} \ln h$ and the posterior regret is

$$
r_{x}(\Pi, a)=\varrho_{x}(h, a)=h e^{-c a}-\ln h+c a-1 .
$$

We have

$$
\frac{\partial \varrho_{x}(h, a)}{\partial h}=e^{-c a}-\frac{1}{h} .
$$

Thus

$$
\sup _{\Pi \in \Gamma} r_{x}(\Pi, a)= \begin{cases}\max \left(\varrho_{x}(\underline{h}, a), \varrho_{x}(\bar{h}, a)\right) & \text { if } a \leq a \leq \bar{a}, \\ \varrho_{x}(\bar{h}, a) & \text { if } a \leq \underline{a}, \\ \varrho_{x}(\underline{h}, a) & \text { if } a \geq \bar{a},\end{cases}
$$

where $\bar{h}=e^{c \bar{a}}$ and $\underline{h}=e^{c \underline{a}}$.

If $a>\bar{a}$ then the function

$$
f_{1}(a)=\varrho_{x}(\underline{h}, a)=\underline{h} e^{-c a}+c a-\ln \underline{h}-1
$$

is increasing $\left(f_{1}^{\prime}(a)=-\underline{h} c e^{-c a}+c>0\right.$ for $\left.a>\underline{a}\right)$. Hence

$$
\inf _{a \geq \bar{a}} f_{1}(a)=f_{1}(\bar{a})=e^{-c(\bar{a}-\underline{a})}+c(\bar{a}-\underline{a})-1
$$

and $f_{1}(\underline{a})=0$.

Similarly the function

$$
f_{2}(a)=\varrho_{x}(\bar{h}, a)=\bar{h} e^{-c a}-\ln \bar{h}+c a-1
$$

is decreasing for $a<\bar{a}$, hence

$$
\inf _{a \leq \underline{a}} f_{2}(a)=f_{2}(\underline{a})=e^{c(\bar{a}-\underline{a})}-c(\bar{a}-\underline{a})-1
$$

and $f_{2}(\bar{a})=0$.

The function $l(a)=f_{1}(a)-f_{2}(a)$ is a continuous function of $a$ in the interval $[\underline{a}, \bar{a}]$ and $l(\underline{a})<0$ and $l(\bar{a})>0$. Thus the properties of $f_{1}$ and $f_{2}$ show that $\widehat{\theta}_{\mathrm{PR}}$ belongs to the interval $(\underline{a}, \bar{a})$ and it is a solution of the equation $f_{1}(a)=f_{2}(a)$. 
Note that if the set $\left\{\widehat{\theta}^{\Pi}(x): \Pi \in \Gamma\right\}$ is connected for every value $x$ of $X$ and the assumptions of Theorem 1 are satisfied, then for every $x$ there exists $\Pi \in \Gamma$ such that $\widehat{\theta}_{\mathrm{PR}}(x)=\widehat{\theta}^{\Pi}(x)$.

\section{Posterior regret $\Gamma$-minimax estimation in a normal model.}

Let $X_{1}, \ldots, X_{n}$ be i.i.d. random variables with the normal $N\left(\theta, b^{2}\right)$ distribution, where $\theta$ is unknown and $b^{2}$ is known. Write $X=\left(X_{1}, \ldots, X_{n}\right)$. Let $\Pi_{\mu, \sigma}=N\left(\mu, \sigma^{2}\right)$ be a prior distribution of $\theta$.

Define

$$
\begin{gathered}
\bar{X}=\frac{1}{n} \sum_{i=1}^{n} X_{i}, \quad V_{n}=V_{n}(\mu)=\frac{n(\bar{X}-\mu)}{b^{2}}, \quad \lambda=\lambda(\sigma)=\left(\frac{1}{\sigma^{2}}+\frac{n}{b^{2}}\right)^{-1}, \\
m=m(\mu, \sigma)=\mu\left(1-\frac{n}{b^{2}}\left(\frac{1}{\sigma^{2}}+\frac{n}{b^{2}}\right)^{-1}\right), \\
W_{n}=W_{n}(\sigma)=\left(\frac{c}{2}+\frac{n \bar{X}}{b^{2}}\right)\left(\frac{1}{\sigma^{2}}+\frac{n}{b^{2}}\right)^{-1} .
\end{gathered}
$$

If $X=x$ then the posterior distribution is the normal distribution

$$
N\left(\mu+v_{n} \lambda, \lambda\right)=N\left(m+w_{n}-\frac{c}{2} \lambda, \lambda\right)
$$

and

$$
E_{\Pi_{\mu, \sigma}}\left(e^{c \theta} \mid x\right)=\exp \left(c \mu+\left(\frac{c^{2}}{2}+c v_{n}\right) \lambda\right)=\exp \left(c m+c w_{n}\right),
$$

and the Bayes estimator is as follows:

$$
\widehat{\theta}^{\mu, \sigma}=\mu+\left(\frac{c}{2}+v_{n}\right) \lambda=m+w_{n},
$$

where $v_{n}, w_{n}$ are the values of the statistics $V_{n}$ and $W_{n}$ for $x$.

Consider two classes of prior distributions of $\theta$, which express two types of uncertainty about the elicited fixed prior $\Pi_{\mu_{0}, \sigma_{0}}$ :

$$
\Gamma_{\mu_{0}}=\left\{\Pi_{\mu_{0}, \sigma}: \Pi_{\mu_{0}, \sigma}=N\left(\mu_{0}, \sigma^{2}\right), \sigma \in\left[\sigma_{1}, \sigma_{2}\right]\right\},
$$

where $\sigma_{1}<\sigma_{2}$ are fixed and $\sigma_{0} \in\left(\sigma_{1}, \sigma_{2}\right)$,

$$
\Gamma_{\sigma_{0}}^{*}=\left\{\Pi_{\mu, \sigma_{0}}: \Pi_{\mu, \sigma_{0}}=N\left(\mu, \sigma_{0}^{2}\right), \mu \in\left[\mu_{1}, \mu_{2}\right]\right\},
$$

where $\mu_{1}<\mu_{2}$ are fixed and $\mu_{0} \in\left(\mu_{1}, \mu_{2}\right)$.

Observe that $\lambda$ is an increasing function of $\sigma$ and therefore if $\sigma \in\left[\sigma_{1}, \sigma_{2}\right]$ then $\lambda \in\left[\lambda_{1}, \lambda_{2}\right]$, where $\lambda_{i}=\lambda\left(\sigma_{i}\right), i=1,2$. Similarly, $m$ is an increasing function of $\mu$ and therefore if $\mu \in\left[\mu_{1}, \mu_{2}\right]$ then $\widetilde{m}=m\left(\mu, \sigma_{0}\right) \in\left[m_{1}, m_{2}\right]$, where $m_{i}=m\left(\mu_{i}, \sigma_{0}\right), i=1,2$. Set $V_{n}^{0}=V_{n}\left(\mu_{0}\right)$ and $W_{n}^{0}=W_{n}\left(\sigma_{0}\right)$. The 
posterior regret can be expressed by two formulas as a function of $\lambda$ and $\widetilde{m}$ :

$$
\begin{aligned}
& r_{x}\left(\Pi_{\mu_{0}, \sigma}, a\right)=\varrho_{x}\left(\mu_{0}, \lambda, a\right) \\
& \quad=\exp \left(-c a+c \mu_{0}\left(\frac{c^{2}}{2}+c v_{n}^{0}\right) \lambda\right)-\left(c \mu_{0}+\left(\frac{c^{2}}{2}+c v_{n}^{0}\right) \lambda\right)+c a-1, \\
& r_{x}\left(\Pi_{\mu, \sigma_{0}}, a\right)=\varrho_{x}^{*}\left(\sigma_{0}, \tilde{m}, a\right)=\exp \left(-c a+c\left(\tilde{m}+w_{n}^{0}\right)\right)-c\left(\widetilde{m}+w_{n}^{0}\right)+c a-1 .
\end{aligned}
$$

THEOREM 2. If the class of priors is equal to $\Gamma_{\sigma_{0}}^{*}$ then

(1) the posterior regret $\Gamma$-minimax estimator is

$$
\widehat{\theta}_{\mathrm{PR}}=m_{1}+w_{n}^{0}+\frac{1}{c} \ln \frac{e^{c\left(m_{2}-m_{1}\right)}-1}{c\left(m_{2}-m_{1}\right)} ;
$$

(2) the estimator $\widehat{\theta}_{\mathrm{PR}}$ is the Bayes estimator with respect to the prior $\Pi_{\mu^{*}, \sigma_{0}} \in \Gamma_{\sigma_{0}}^{*}$, where $\mu^{*}$ satisfies

$$
\mu^{*}=\mu_{1}+\frac{1}{c z} \ln \frac{e^{c z\left(\mu_{2}-\mu_{1}\right)}-1}{c z\left(\mu_{2}-\mu_{1}\right)}
$$

and

$$
z=1-\frac{n}{b^{2}}\left(\frac{1}{\sigma_{0}^{2}}+\frac{n}{b^{2}}\right)^{-1}
$$

(3) the estimator $\widehat{\theta}_{\mathrm{PR}}$ is the most stable and conditional $\Gamma$-minimax.

Proof. The function $E_{\Pi_{\mu, \sigma_{0}}}\left(e^{c \theta} \mid x\right)=\exp \left(c\left(\widetilde{m}+w_{n}^{0}\right)\right)$ is an increasing function of $\widetilde{m}$, hence

$$
\inf _{\Pi \in \Gamma_{\sigma_{0}}^{*}} \widehat{\theta}^{\mu, \sigma_{0}}=m_{1}+w_{n}^{0}, \quad \sup _{\Pi \in \Gamma_{\sigma_{0}}^{*}} \widehat{\theta}^{\mu, \sigma_{0}}=m_{2}+w_{n}^{0} .
$$

Now applying Theorem 1 we obtain $\widehat{\theta}_{\mathrm{PR}}$.

To prove (2) find $\mu^{*} \in\left[\mu_{1}, \mu_{2}\right]$ such that for almost all values $x$,

$$
m_{1}+w_{n}^{0}+\frac{1}{c} \ln \frac{e^{c\left(m_{2}-m_{1}\right)}-1}{c\left(m_{2}-m_{1}\right)}=m\left(\mu^{*}, \sigma_{0}\right)+w_{n}^{0} .
$$

Substituting the definition of $m$ we obtain $\mu^{*}$.

For (3) see Boratyńska and Drozdowicz [5].

Note that $\mu^{*}$ does not depend on the value of the random variable $X$. Thus if the uncertainty of prior elicitation is expressed by the class $\Gamma_{\sigma_{0}}^{*}$ then using the Bayes estimator with respect to the normal $N\left(\mu^{*}, \sigma_{0}^{2}\right)$ distribution we obtain the best estimator with respect to three criteria of robustness.

THEOREM 3. If the class of priors is equal to $\Gamma_{\mu_{0}}$ then the posterior regret $\Gamma$-minimax estimator is

$$
\widehat{\theta}_{\mathrm{PR}}=\mu_{0}+\left(\frac{c}{2}+v_{n}^{0}\right) \lambda_{1}+\frac{1}{c} \ln \frac{\exp \left(\left(c^{2} / 2+c v_{n}^{0}\right)\left(\lambda_{2}-\lambda_{1}\right)\right)-1}{\left(c^{2} / 2+c v_{n}^{0}\right)\left(\lambda_{2}-\lambda_{1}\right)}
$$


for $v_{n}^{0} \neq-c / 2$. For $v_{n}^{0}=-c / 2$ the posterior regret does not depend on $\lambda$ and $\widehat{\theta}_{\mathrm{PR}}=\mu_{0}$.

Proof. Consider the Bayes estimator

$$
\widehat{\theta}^{\mu_{0}, \sigma}=t(\lambda, x)=\mu_{0}+\left(c / 2+v_{n}^{0}\right) \lambda .
$$

If $v_{n}^{0}>-c / 2$ (resp. $v_{n}^{0}<-c / 2$ ) then $t$ is an increasing (resp. a decreasing) function of $\lambda$ and applying Theorem 1 we obtain $\widehat{\theta}_{\mathrm{PR}}$. If $v_{n}^{0}=-c / 2$ then $t(\lambda, x)=\mu_{0}$ and the posterior regret is

$$
r_{x}\left(\Pi_{\mu_{0}, \sigma}, a\right)=\exp \left(c\left(\mu_{0}-a\right)\right)-c\left(\mu_{0}-a\right)-1 .
$$

The minimum of the function $r_{x}$ is attained for $a=\mu_{0}$.

Note that if the class of priors is $\Gamma_{\mu_{0}}$ then

(i) $\widehat{\theta}_{\mathrm{PR}}$ is neither the most stable nor the conditional $\Gamma$-minimax estimator (see Boratyńska and Drozdowicz [5]).

(ii) For every value $x$ of the random variable $X$ there exists a prior $\Pi_{\mu_{0}, \sigma^{*}} \in \Gamma_{\mu_{0}}$ such that $\widehat{\theta}_{\mathrm{PR}}(x)=\widehat{\theta}^{\mu_{0}, \sigma^{*}}(x)$. For $v_{n}^{0} \neq-c / 2$ the parameter $\sigma^{*}$ satisfies the condition

$$
\lambda\left(\sigma^{*}\right)=\lambda_{1}+\frac{1}{c z} \ln \frac{e^{c z\left(\lambda_{2}-\lambda_{1}\right)}-1}{c z\left(\lambda_{2}-\lambda_{1}\right)},
$$

where $z=c / 2+v_{n}^{0}$, and $\sigma^{*}$ depends on the value of the random variable $X$.

Note that the condition $v_{n}^{0}=-c / 2$ is equivalent to the condition that a sample mean is equal to $-b^{2} c /(2 n)+\mu_{0}$ and the probability of this event is 0 .

\section{References}

[1] J. Berger, The robust Bayesian viewpoint, in: Robustness of Bayesian Analyses, J. Kadane (ed.), Elsevier, Amsterdam, 1984, 63-124.

[2] B. Betro and F. Ruggeri, Conditional $\Gamma$-minimax actions under convex losses, Comm. Statist. Theory Methods 21 (1992), 1051-1066.

[3] A. Boratyńska, Stability of Bayesian inference in exponential families, Statist. Probab. Lett. 36 (1997), 173-178.

[4] -, Robust Bayesian estimation with asymmetric loss function, Appl. Math. (Warsaw), to appear.

[5] A. Boratyńska and M. Drozdowicz, Robust Bayesian estimation in a normal model with asymmetric loss function, ibid. 26 (1999), 85-92.

[6] M. Męczarski, Stability and conditional $\Gamma$-minimaxity in Bayesian inference, ibid. 22 (1993), 117-122.

[7] M. Męczarski and R. Zieliński, Stability of the Bayesian estimator of the Poisson mean under the inexactly specified gamma prior, Statist. Probab. Lett. 12 (1991), 329-333. 
[8] D. Rios Insua, F. Ruggeri and B. Vidakovic, Some results on posterior regret $\Gamma$ minimax estimation, Statist. Decisions 13 (1995), 315-331.

[9] B. Vidakovic, $\Gamma$-minimax: a paradigm for conservative robust Bayesians, in: Lecture Notes in Statist. 152, Springer, New York, 2000, 241-259.

[10] A. Wan, G. Zou and A. Lee, Minimax and $\Gamma$-minimax estimation for the Poisson distribution under LINEX loss when the parameter space is restricted, Statist. Probab. Lett. 50 (2000), 23-32.

Institute of Econometrics

Warsaw School of Economics

Al. Niepodległości 162

02-554 Warszawa, Poland

E-mail: aborata@sgh.waw.pl

Received on 7.11.2001;

revised version on 6.2.2002 